\title{
RELACIONES QUE ESTABLECEN LAS MUJERES DURANTE EL EMBARAZO, PARTO Y POSPARTO CON EL PERSONAL DE SALUD SEGÚN CLASE SOCIAL EN BOGOTÁ: ESTUDIO CUALITATIVO
}

\author{
Libia A. Bedoya-Ruiz (10), Andrés A. Agudelo-Suárez (10) 1,2,b, Diego A. Restrepo-Ochoa®1],3,c \\ Escuela de Graduados, Universidad CES, Medellín, Colombia. \\ 2 Facultad de Odontología, Universidad de Antioquia, Medellín, Colombia. \\ 3 Facultad de Psicología, Universidad CES, Medellín, Colombia. \\ Médico, magíster en Salud Pública; ${ }^{b}$ odontólogo, doctor en Salud Pública; ${ }^{c}$ psicólogo, doctor en Salud Pública.
}

Esta investigación es un producto académico de: Bedoya Ruiz LA. Configuración de la relación de las mujeres en embarazo, Parto y Postparto con los servicios de salud en diferentes niveles de atención según la clase social. Bogotá (Colombia). [Tesis de doctorado]. Medellín: Escuela de Graduados, Universidad CES de Medellín; 2020.

\section{RESUMEN}

Objetivo: Comprender la relación que se establece entre las mujeres en situación de embarazo, parto y posparto con el personal de los servicios de salud según la clase social en Bogotá (Colombia). Materiales y métodos: Estudio cualitativo, perspectiva hermenéutica crítica y etnografía crítica con muestreo teórico. Análisis por triangulación en Atlas.ti. Participaron nueve mujeres y ocho profesionales de la salud. Se realizaron 38 entrevistas a profundidad durante 13 meses y 62 acompañamientos a las mujeres en las actividades de control prenatal, vacunación, trabajo de parto, consulta posparto, exámenes de seguimiento, curso psicoprofiláctico, hospitalización y sala de espera, tanto en servicios públicos como privados. Resultados: Existen desigualdades según la clase social en la forma en que se configura la relación entre las mujeres y el personal en los siguientes aspectos: permeabilidad a las necesidades de la mujer, reconocimiento de aspectos psicosociales, tener diferentes puntos de vista frente a una recomendación médica y derecho a quejarse o exigir para mejorar la atención de los servicios de salud. Conclusiones: Lo anterior intensifica las problemáticas relacionadas con el género en las mujeres con una clase social menos aventajada. Es necesario el desarrollo de intervenciones en las instituciones educativas y de salud que tengan en cuenta aspectos donde se sensibilice el recurso humano en temas sociales relacionados con las propuestas teóricas de la investigación y la democratización de la información médica. Es injusto que la condición de clase social y de género afecte la calidad en la atención y estratifique económicamente los derechos de las personas.

Palabras clave: Clase Social; Relaciones Interpersonales; Embarazo; Parto; Periodo Posparto (fuente: DeCS BIREME).

Citar como: Bedoya-Ruiz LA, Agudelo-Suárez AA, Restrepo-Ochoa DA. Relaciones que establecen las mujeres durante el embarazo, parto y posparto con el personal de salud según clase social en Bogotá: estudio cualitativo. Rev Peru Med Exp Salud Publica. 2020;37(1): 7-16. Doi: https://doi.org/10.17843/ rpmesp.2020.371.4963

Correspondencia: Libia A. BedoyaRuiz; Escuela de Graduados, Universidad CES, Medellín, Colombia; Calle 10 A No. 22 - 04. Medellín, Colombia; bedoya.libia@ces.edu.co, bedoyalibia@hotmail.com

Recibido: $14 / 11 / 2019$

Aprobado: $05 / 02 / 2020$

En línea: 23/03/2020

\section{RELATIONS ESTABLISHED BY WOMEN DURING PREGNANCY, DELIVERY AND POSTPARTUM WITH HEALTH PERSONNEL ACCORDING TO SOCIAL CLASS IN BOGOTÁ: QUALITATIVE STUDY}

\section{ABSTRACT}

Objective: To understand the relationship established between women in a situation of pregnancy, childbirth and postpartum with the health services personnel according to social class in Bogotá (Colombia). Materials and methods: Qualitative study. Critical hermeneutical perspective and critical ethnography. Theoretical sampling. Analysis by triangulation in Atlas.ti. 9 women and 8 health professionals participated. 38 in-depth interviews were conducted for 13 months and 62 accompaniments to the maternal in the activities of prenatal control, vaccination, labor, postpartum consultation, follow-up exams, prophylactic pisco course, hospitalization and waiting room, both in public services as private. Results: There are inequalities according to social class in which the relationship between women and staff is configured in the following aspects: permeability to the needs of women, recognition of psychosocial aspects, having different points of view against a medical recommendation and right to complain or demand to improve. Conclusions: The situation described above intensifies gender issues in women with a less advantageous social class. It is necessary to develop interventions in educational and health institutions that consider aspects where human resources are sensitized on social issues related to the theoretical proposals of research and the democratization of medical information. It is unfair that the condition of social class and gender affects the quality of care and economically stratifies people's rights.

Keywords: Social Class; Interpersonal Relations; Pregnancy; Childbirth; Postpartum Period (source: MeSH NLM). 


\section{INTRODUCCIÓN}

Mundialmente se ha evidenciado situaciones de maltrato a las mujeres en embarazo, parto y posparto (EPP) por parte del personal de salud ${ }^{(1,2)}$. Aunque se han intentado introducir programas en salud pública para implementar un cuidado respetuoso en la maternidad, los efectos de estas intervenciones no han sido satisfactorios en todos los casos. Por ejemplo, aunque el abuso físico se redujo, otras situaciones no disminuyeron, como el abuso verbal, la negligencia y el abandono ${ }^{(1)}$.

La relación entre las mujeres en EPP y el personal también es de interés en Colombia, donde se han desarrollado investigaciones que muestran la presencia de violencia obstétrica y de género ${ }^{(3-8)}$. Si bien los anteriores estudios toman mujeres de diferentes niveles educativos, no alcanzan a visibilizar las desigualdades en las relaciones según la clase social. Esto muestra vacíos conceptuales y metodológicos en la investigación de salud pública colombiana sobre este tema ${ }^{(9)}$, haciendo necesario no solo profundizar en los indicadores de morbimortalidad materna, sino también en la calidad de la atención en salud.

Concretamente este estudio se plantea desde las teorías propuestas por Breilh y Menéndez ${ }^{(10-13)}$. El primero tiene una posición neomarxista, la cual explica que la clase social no es un asunto de elección personal, sino que está determinada por aspectos estructurales. Esto permite comprender que las desigualdades en salud son reflejo de desigualdades sociales. En este sentido la clase social se comprende como un grupo de personas que tienen intereses en común y se diferencian en el poder que se tiene en las jerarquías del proceso de trabajo, lo cual determina la capacidad de acumular riqueza ${ }^{(10,11)}$.

Por otro lado, Menéndez se enfoca en la Antropología Médica Crítica, la cual no se centra únicamente en las diferencias culturales (vistas como barrera para el diálogo de saberes entre el sistema biomédico y el sistema médico popular), dado que también es necesario incluir aspectos relacionados con la pobreza y las desigualdades sociales ${ }^{(12,13)}$. El concepto de modelo médico hegemónico no solo excluye ideológicamente los saberes construidos históricamente por los sujetos, sino que también ignora a las personas más pobres y los colectivos étnicos ${ }^{(12,13)}$.

Teniendo en cuenta lo anterior, este estudio tuvo como objetivo comprender la relación que se establece entre las mujeres en situación de embarazo, parto y posparto con el personal de los servicios de salud según la clase social en la ciudad de Bogotá (Colombia).

\section{MATERIALES Y MÉTODOS}

Se realizó un estudio cualitativo, flexible ${ }^{(18,19)}$, con enfoque teórico-metodológico que se transformó de acuerdo a lo en-

\section{MENSAJES CLAVE}

Motivación para realizar el estudio: Es importante evidenciar los vacíos existentes en la literatura, teniendo en cuenta que las mujeres en embarazo, parto y posparto son obviadas como sujetos en frecuentes ocasiones por los profesionales de la salud.

Principales hallazgos: Existen desigualdades en la relación que establecen las mujeres con el personal de salud según la clase social.

Implicancias: Se deben buscar alternativas en el proceso de atención que trasciendan al modelo biomédico tradicional. En las políticas sanitarias se requieren transformaciones importantes en el sistema de educación médica y de salud.

contrado en el trabajo de campo, y fue descrito en un documento de memoria metodológica ${ }^{(19)}$, revisado por el grupo de investigación y evaluadores externos ajenos al proceso investigativo (confiabilidad y auditabilidad).

\section{Fases del estudio y participantes}

La exploración empezó en mayo de 2017. El trabajo de campo empezó en noviembre de 2017 y finalizó en diciembre de 2018. En esta fase se hizo presencia en el terreno y se realizaron intercambios virtuales en las redes sociales que construyó sintonía con los participantes para generar un ambiente de confianza donde los sujetos pudieron expresar libremente lo que pensaban en el momento que ellos deseaban. La fase de análisis y redacción de la información inició en el trabajo de campo y finalizó en noviembre de 2019.

En este periodo de estudio, participaron nueve mujeres de la ciudad de Bogotá, en primer trimestre de embarazo o más, y ocho profesionales de la salud cuyas características sociodemográficas se resumen en la Tabla 1 y 2 . No participaron mujeres con enfermedad mental, discapacidad, menores de edad y quienes pudieran cambiar de residencia. Las mujeres y el personal fueron contactados a través de redes sociales y el servicio de control prenatal del sector público, tres mujeres rechazaron participar pues no requerían el acompañamiento propuesto.

\section{Procedimiento}

\section{Perspectiva epistemológica}

Se tuvo en cuenta la hermenéutica crítica que incluye elementos de la hermenéutica y de la teoría crítica. Gadamer explica que la interpretación de la hermenéutica no es «una reproducción de una producción original», es una mediación entre el investigador y el investigado y por lo tanto se requiere de la comprensión, la cual se aleja de la objetividad. Las preconcepciones son construidas por aprendizajes de 
Tabla 1. Características sociodemográficas de mujeres gestantes participantes en la investigación. Bogotá

\begin{tabular}{|c|c|c|c|c|c|c|c|c|c|c|}
\hline $\begin{array}{l}\text { Mujer par- } \\
\text { ticipante }\end{array}$ & $\begin{array}{l}\text { Régimen } \\
\text { salud }\end{array}$ & $\begin{array}{l}\text { Edad } \\
\text { (años) }\end{array}$ & $\begin{array}{l}\text { Semanas } \\
\text { gestación } \\
\text { inicio } \\
\text { estudio }\end{array}$ & Ocupación & Nivel educativo & $\begin{array}{l}\text { Estado } \\
\text { civil }\end{array}$ & $\mathrm{ESE}^{\mathrm{a}}$ & $\begin{array}{c}\text { Fuente } \\
\text { ingresos }\end{array}$ & $\begin{array}{l}\text { Localidad } \\
\text { vivienda }\end{array}$ & $\begin{array}{l}\text { Localidad } \\
\text { servicios } \\
\text { salud }\end{array}$ \\
\hline Gp & Prepagada & 38 & 27 & $\begin{array}{l}\text { Empresar- } \\
\text { ia servicios } \\
\text { privados me- } \\
\text { dicina estética }\end{array}$ & $\begin{array}{l}\text { Profesional } \\
\text { Medicina } \\
\text { posgrado }\end{array}$ & Casada & 5 & $\begin{array}{l}\text { Trabajo (de } \\
\text { ella, pareja) y } \\
\text { propiedades }\end{array}$ & Usaquén & Usaquén \\
\hline Ap & $\begin{array}{c}\text { Plan complemen- } \\
\text { tario } \mathrm{C}^{\mathrm{b}}\end{array}$ & 32 & 8 & Ama de casa & $\begin{array}{l}\text { Profesional } \\
\text { Ingeniería }\end{array}$ & Casada & 3 & $\begin{array}{c}\text { Trabajo de } \\
\text { pareja y propie- } \\
\text { dades }^{\mathrm{c}}\end{array}$ & $\begin{array}{l}\text { Cercanía } \\
\text { Suba }\end{array}$ & Chapinero \\
\hline Lp & $\begin{array}{c}\text { Plan complemen- } \\
\text { tario S }\end{array}$ & 34 & 32 & $\begin{array}{c}\text { Trabajo infor- } \\
\text { mal investiga- } \\
\text { dora }\end{array}$ & $\begin{array}{l}\text { Profesional } \\
\text { Antropología } \\
\text { posgrado }\end{array}$ & $\begin{array}{l}\text { Unión } \\
\text { marital de } \\
\text { hecho }\end{array}$ & 3 & $\begin{array}{l}\text { Trabajo de ella, } \\
\text { pareja, padre }\end{array}$ & Engativá & $\begin{array}{l}\text { Chapinero, } \\
\text { Usaquén }\end{array}$ \\
\hline $\mathrm{Kc}$ & Contributivo S & 27 & 28 & Asalariada & $\begin{array}{l}\text { Profesional } \\
\text { Ingeniería }\end{array}$ & Casada & 3 & $\begin{array}{c}\text { Trabajo de ella, } \\
\text { pareja }\end{array}$ & Kennedy & $\begin{array}{l}\text { Kennedy, } \\
\text { Teusaquillo }\end{array}$ \\
\hline Ac & Contributivo $\mathrm{N}$ & 23 & 16 & Asalariada & $\begin{array}{c}\text { Técnica en } \\
\text { gestión de ar- } \\
\text { chivo }\end{array}$ & Casada & 2 & $\begin{array}{c}\text { Trabajo de ella, } \\
\text { pareja }\end{array}$ & Bosa $^{f}$ & $\begin{array}{l}\text { Bosa }{ }^{\mathrm{f}} \text {, Chap- } \\
\text { inero } \\
\text { Puente Aran- } \\
\text { da }\end{array}$ \\
\hline $\mathrm{Lc}$ & Contributivo C & 18 & 20 & Ama de casa & Bachiller & Soltera & 2 & $\begin{array}{l}\text { Trabajo de pa- } \\
\text { dres, hermanos }\end{array}$ & Bosa $^{f}$ & $\begin{array}{c}\text { Kennedy } \\
\text { Usaquén } \\
\text { Puente Aran- } \\
\text { da }\end{array}$ \\
\hline Ss & Subsidiado $\mathrm{ES}^{\mathrm{d}}$ & 19 & 25 & Ama de casa & $\begin{array}{l}\text { Séptimo bachil- } \\
\text { lerato }\end{array}$ & $\begin{array}{c}\text { Unión } \\
\text { marital de } \\
\text { hecho }\end{array}$ & 1 & $\begin{array}{c}\text { Trabajo de } \\
\text { pareja, suegra }\end{array}$ & $\begin{array}{l}\text { Ciudad } \\
\text { Bolívar }^{\mathrm{f}}\end{array}$ & $\begin{array}{l}\text { Ciudad } \\
\text { Bolívar }^{\mathrm{f}} \\
\text { Tunjuelito }^{\mathrm{f}}\end{array}$ \\
\hline Gs & Subsidiado ES & 36 & 20 & $\begin{array}{c}\text { Trabajo infor- } \\
\text { mal varios }\end{array}$ & $\begin{array}{c}\text { Octavo bachil- } \\
\text { lerato }\end{array}$ & Soltera & 1 & Trabajo de ella & Bosa $^{\mathrm{f}}$ & Bosa $^{\mathrm{f}}$ \\
\hline Vs & Subsidiado $\mathrm{FF}^{\mathrm{e}}$ & 27 & 38 & $\begin{array}{c}\text { Trabajo infor- } \\
\text { mal }\end{array}$ & Bachiller & Soltera & 1 & $\begin{array}{l}\text { Trabajo de } \\
\text { padres }\end{array}$ & $\begin{array}{l}\text { Ciudad } \\
\text { Bolívar }^{\mathrm{f}}\end{array}$ & $\begin{array}{l}\text { Ciudad } \\
\text { Bolívar }^{\mathrm{f}}\end{array}$ \\
\hline
\end{tabular}

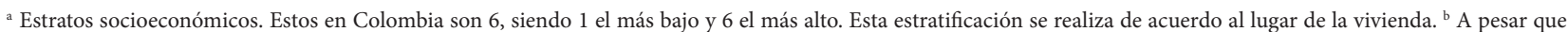
dos mujeres tienen planes complementarios las empresas que prestan los servicios son diferentes y por lo tanto las clínicas de atención son diferentes. Lo anterior también sucede en el régimen contributivo y subsidiado. Por lo tanto, se coloca la inicial para visibilizar las empresas que son diferentes. ${ }^{c}$ Familias que tienen capacidad de ahorro y reciben de ingresos de otras fuentes diferentes al trabajo (arriendos, alquiler de equipos). ${ }^{\mathrm{d}}$ EPS subsidiada. ${ }^{\mathrm{e}}$ Fondo financiero. ${ }^{\mathrm{f}}$ Localidades del sur de Bogotá vulnerables con peores indicadores de salud materno fetal, contaminación ambiental, déficit de transporte público, inseguridad, déficit de servicios de salud, traslados prolongados entre el domicilio y los servicios de salud, servicios de salud públicos involucrados en corrupción.

vida y esto determina una manera de interpretar el sujeto ${ }^{(14)}$. Habbermas integra la interpretación con la teoría crítica que requiere objetividad para analizar las condiciones materiales de la población. Este autor argumenta que las preconcepciones no siempre son legítimas pues las tradiciones pueden ser impuestas, la interpretación debe servir para criticar la ideología, pues aunque es necesario comprender el punto de vista del actor, también es relevante develar relaciones de poder que permitan desarrollar emancipación ${ }^{(15)}$. Lo anterior da cuenta de la epistemología de la complejidad donde los investigadores «bricoleurs» no se limitan a la producción de significados de la hermenéutica pura, pues se puede generar resistencia a la trasformación de las condiciones sociales ${ }^{(16)}$.

\section{Método}

Etnografía crítica desde el enfoque relacional de Menéndez que vincula aspectos microsociales (relaciones entre sujetos) y macrosociales (condiciones estructurales) ${ }^{(13)}$, y la postura de Sheper-Hughes con respecto al rol del investigador, quien no intenta mimetizarse entre la población, sino que hace conciencia de que su presencia en la comunidad va a transformar su vida y la de los sujetos de estudio, por eso cuestiona de manera respetuosa y crítica las situaciones de salud que la población normaliza, siendo aspectos que contribuyen a la opresión en medio de la pobreza y desigualdad. Pero este diálogo es bilateral, pues permite igualmente que la población la cuestione y se aprende de esto también ${ }^{(17)}$.

\section{Criterios de calidad}

Se evaluó la credibilidad y confiabilidad ${ }^{(13,18,19)}$, teniendo en cuenta la participación de la investigadora principal que puede cambió el comportamiento de los sujetos. En el desarrollo del trabajo de campo, se evidenciaron situaciones donde se afectaban los derechos sexuales y reproductivos de las mu- 
Tabla 2. Características del personal de salud con experiencia en atención materna participante del estudio, Bogotá

\begin{tabular}{|c|c|c|c|c|}
\hline Tipo & Lugar de trabajo & Sexo & $\begin{array}{c}\text { Edad } \\
\text { (años) }\end{array}$ & $\begin{array}{l}\text { Experiencia laboral } \\
\text { en maternidad (años) }\end{array}$ \\
\hline Obstetra atención de partos, consulta externa, preparación parto & Prepagada & $\mathrm{F}$ & 48 & 18 \\
\hline Asesora de lactancia materna & Prepagada y contributivo & $\mathrm{F}$ & 41 & 21 \\
\hline Enfermera consulta externa & Prepagada y contributivo & $\mathrm{F}$ & 28 & 4 \\
\hline Medica atención de partos, consulta externa & Contributivo y subsidiado & $\mathrm{F}$ & 40 & 5 \\
\hline Obstetra consulta externa & Subsidiado & M & 54 & 25 \\
\hline Obstetra atención de partos, consulta externa & Subsidiado & $\mathrm{F}$ & 32 & 8 \\
\hline $\begin{array}{l}\text { Enfermera consulta externa y preparación embarazo, parto y } \\
\text { posparto }\end{array}$ & Subsidiado & $\mathrm{F}$ & 46 & 15 \\
\hline $\begin{array}{l}\text { Fisioterapeuta atención de partos, preparación embarazo, parto } \\
\text { y posparto }\end{array}$ & Contributivo y prepagada & $\mathrm{F}$ & 33 & 5 \\
\hline
\end{tabular}

F: Femenino, M: Masculino

jeres. En algunos casos, las participantes naturalizaban esta situación y no visibilizaban la problemática, en otros casos buscaban ayuda en la investigadora. Para ambos casos, la participación de la investigadora principal consistió en entregar de información documentada relacionada con los derechos, apoyar para clarificar términos médicos, formular preguntas sobre sus deseos y oportunidades, y contactar redes de apoyo relacionadas con la salud sexual y reproductiva, e instituciones estatales que vigilan los servicios de salud. Todo lo anterior se realizó con el fin de que las mujeres tuvieran insumos para construir autonomía en la toma de decisiones y fueran ellas y no la investigadora quienes transformaran las situaciones ocurridas en los servicios de salud, lo cual es coherente con el paradigma epistemológico elegido. El marco referencial se construyó a lo largo del proceso investigativo, lo que permitió, al final de la investigación, analizar los resultados desde diferentes perspectivas teóricas (credibilidad).

Se diferenció en el proceso de redacción, los discursos de los actores de los análisis realizados por la investigadora. El registro de la información quedó a disposición de evaluadores externos que deseen verificar los resultados encontrados (confiablidad y auditabilidad).

\section{Muestreo}

De tipo teórico ${ }^{(19)}$, teniendo en cuenta los conceptos de clase social de Breilh ${ }^{(10,11)}$ y el tipo de afiliación en salud en Colombia ${ }^{(20)}$. Se diferenciaron tres categorías de acuerdo con la situación laboral relacionada con la clase social ${ }^{(21)}$ : régimen subsidiado (mujeres con trabajo informal, población sin capacidad de pago), régimen contributivo básico (mujeres asalariadas), y régimen contributivo prepagado o planes complementarios (mujeres asalariadas o empresarias con capacidad de pago de servicios privados).

\section{Estrategias y técnicas metodológicas}

Se realizaron de tres a cinco entrevistas a profundidad por mujer durante el EPP (con recién nacido vivo) y se recopiló información en diferentes momentos del proceso investigativo (credibilidad). Contando el personal sanitario, fueron en total 38 entrevistas.
Las entrevistas fueron realizadas por la investigadora principal de manera no estructurada con preguntas abiertas ${ }^{(22)}$. Se utilizó una guía que incluyó temas relacionados con la clase social $^{(9-11)}$ y los significados de la relación de las mujeres con el personal de salud ${ }^{(9,12)}$. Los encuentros se programaron según disponibilidad de los participantes en lugares elegidos por ellos. Las conversaciones tuvieron un carácter privado y confidencial: en las organizaciones sociales, el domicilio cuando las mujeres estaban solas, y restaurantes poco frecuentados. La duración fue en promedio una hora.

La observación participante ${ }^{(13,23)}$ la realizó la investigadora principal en los servicios de salud donde asistieron las mujeres (Tabla 1). Se efectuaron 62 acompañamientos a mujeres durante el embarazo, parto y posparto. Lo observado se registró en el diario de campo donde se tomaron pequeñas notas a diario. Se buscó que no se bloqueara la interacción con los sujetos. La redacción completa se realizó cuando no se estaba en el terreno. Se construyeron itinerarios de acompañamiento en relato etnográfico, con información descriptiva y detallada (credibilidad) que incluyó: punto de vista de cada mujer (visibilización del proceso de EPP en un contexto histórico y situado), la posición de otros sujetos diferentes a los participantes (credibilidad), los diálogos establecidos y la reflexividad de la investigadora. Se recopiló información estadística y epidemiológica con la cual se construyó el contexto de la investigación que ayudó a dar significado a los resultados. Todo lo anterior está disponible en la Escuela de Graduados de la Universidad CES de Medellín para dar cuenta de la transferibilidad a otros contextos urbanos latinoamericanos con sistemas de salud similares.

\section{Análisis cualitativo}

En los itinerarios de acompañamiento se identificaron aspectos que tienen en común los participantes y lo que los diferencian. Está información se organizó en el proceso de EPP lo que permitió ver recurrencias de aspectos teóricos a través del tiempo por cada una de las mujeres ${ }^{(23)}$. Lo anterior se transcribió en un formato Excel y fue un insumo importante para el análisis de las entrevistas pues había aspectos en 
ellas que parecían no recurrentes, pero que al triangularlos (credibilidad) con los itinerarios de acompañamiento adquirían importancia teórica.

Las entrevistas fueron grabadas por la investigadora principal y transcritas por dos transcriptoras de la Universidad CES de Medellín. Las transcripciones de las entrevistas y el itinerario de acompañamiento fueron entregados a los participantes para retroalimentación.

El análisis se realizó en Atlas.ti versión 7, se le asignó un código a los párrafos o frases lo cual permitió clasificar la información. Se buscó desagregar datos, para volverlos a unir de manera diferente en forma de categorías. Esto busca construir conceptos relacionados con la teoría (análisis de contenido) y agrupar datos que tengan significados similares, con el fin de construir conceptos que le den sentido a los resultados ${ }^{(24)}$. Se identificaron 179 códigos derivados de los datos, organizados en 11 categorías. En el presente artículo se publican dos categorías en los resultados. El proceso de codificación se realizó con el grupo de investigación y una trabajadora social.

Se triangularon los resultados teniendo en cuenta las técnicas metodológicas (credibilidad) y el proceso de socialización ${ }^{(18,19)}$. Este se realizó con pares investigadores de la Universidad CES y expertos en el tema, ajenos al proceso, lo que permitió incluir perspectivas diferentes e interdisciplinarias (confiabilidad, auditabilidad). Igualmente la socialización fue progresiva con los participantes para validar la información y proponer estrategias que emerjan de ellos mismos.

\section{Aspectos éticos}

Se contó con aprobación del comité de ética de la Universidad CES (Acta No 99/2016) y de la Secretaria de salud de Bogotá (Acta N0041000/2017). Según el código de ética médica colombiano, se respetó en el ámbito asistencial las decisiones clínicas tomadas por el personal de salud, pues la atención dada por los trabajadores de la salud excluyó la posible atención médica requerida de la investigadora principal. En el consentimiento informado se aclaró quien era la investigadora y sus intereses siendo mujer, médica y madre.

\section{RESULTADOS}

Se encontraron dos categorías (Figuras 1) las cuales se profundizan a continuación.

\section{Relación de confianza entre el personal y las muje- res (Figura 1A)}

Para establecer vínculos entre el personal y las mujeres fue necesaria la confianza. Esta permite establecer un vínculo terapéutico donde las mujeres aceptan las recomendaciones del personal y existe adherencia a las iniciativas de los servicios de salud. La confianza cambia según los aspectos desarrollados en la Tabla 3.

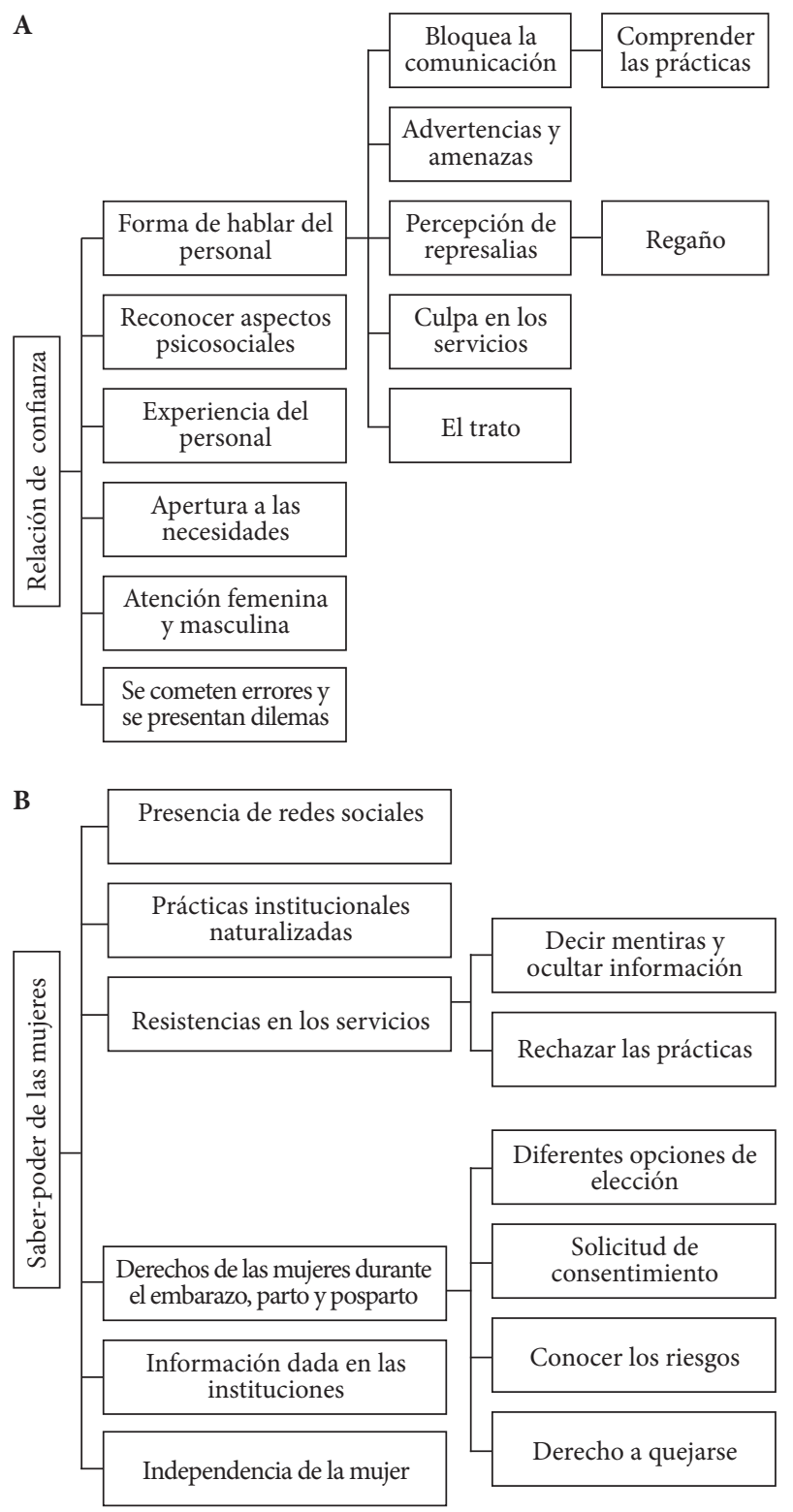

Figura 1. A. Relación de confianza entre el personal y las mujeres. B. El saber-poder de las mujeres

En la observación participante se evidenció que fueron vulnerados varios derechos sexuales y reproductivos en todas las mujeres participantes, lo cual implica que la violencia obstétrica y de género afecta diferentes clases sociales. Sin embargo, para las mujeres con una clase social menos aventajada existen problemas que se intensifican en mayor medida. Por ejemplo, la falta de apertura a las necesidades de las mujeres y la ausencia de reconocimiento de aspectos psicosociales. Al respecto, el personal de salud trata a las participantes como ignorantes y descuidadas con respecto a su proceso de EPP. Las mujeres son regañadas, y juzgadas cuando no se cumple con los protocolos médicos. No se tiene en cuenta el contexto social pues son mujeres que viven en las 
Tabla 3. Códigos de la categoría relación de confianza entre el personal y las mujeres

\begin{tabular}{ll}
\hline Código & \multicolumn{1}{c}{ Definición conceptual } \\
\hline & $\begin{array}{l}\text { Se refiere al tono de la voz, la forma decir las cosas, el } \\
\text { uso de lenguaje técnico, la ausencia de comunicación. } \\
\text { Los aspectos que rompen la relación de confianza y blo- } \\
\text { Forma de hablar }\end{array}$ \\
del personal & $\begin{array}{l}\text { la realización de advertencias, el regaño como castigo. } \\
\text { Los anteriores temas se relacionaron con la percepción } \\
\text { de recibir mal trato, sentirse mal en los servicios y no } \\
\text { comprender ni aceptar las prácticas institucionales }\end{array}$
\end{tabular}

Reconocimiento de aspectos psicosociales

Experiencia que tiene el personal

Se relaciona con la experticia en determinado tema que a la mujer le da seguridad y confianza en el proceso de atención

Incluye la validación de las emociones, el comprender como se sienten y quienes son las mujeres en el mundo en que viven. Este aspecto se relaciona con la capacidad que tiene el personal para colocarse en lugar del otro

Apertura a las necesidades

Es el espacio que tienen las mujeres para participar en el proceso de atención, planteando preguntas e inquietudes. Es el derecho a ser escuchadas sin recibir regaños ni ser juzgadas

Se refiere a los roles de género donde lo femenino está asociado con la empatía, la permeabilidad a las necesiAtención femenina dades de las mujeres y el reconocimiento de aspectos y masculina psicosociales. Lo masculino es la ausencia de todo lo anterior. Estos roles pueden ser asumidos tanto por trabajadoras y como por trabajadores de la salud.

Es la percepción de los usuarios referente a los errores Cometer errores y presentar dilemas en la atención que se presentan en la atención y se visualizan dilemas en los abordajes terapéuticos, pues la medicina no es una ciencia exacta y no siempre es evidente tomar decisiones en salud
Citas textuales

«El doctor dijo: "si, es que para mí como científico es más importante que el corazón está bien, pero como para ustedes es tan importante verle la cara" y yo era como ay que grosero, o sea, puede que sea cierto ¿sí?, pero 1 no tiene que restregarme en la cara su autoridad científica y 2 listo, podría decirme "mira, esto está bien” ¿me entiendes?, pero hacerlo de una manera que no sea tan prepotente». Mujer profesional con especialidad, trabajo informal, plan complementario.

«Es inquietarnos un poco sobre la emocionalidad en el parto, porque como ese capítulo de psicología del embarazo y del parto no lo vimos, todavía conservamos la disociación desde la raíz académica de solamente ver como la parte física. El trato podría ser un poco diferente, así el sistema de salud no haya cambiado». Obstetra régimen subsidiado y prepagada.

«A mí me parecen tan aburridos los cursos psicoprofilácticos...Me parece aburrido porque, yo sé por ejemplo que la persona que dicta el curso psicoprofiláctico nunca ha tenido un hijo...lo sé porque ella lo dijo una vez. Entonces yo digo pues que una persona que nunca ha tenido un hijo, que nunca ha tenido experiencia, que no sabe lo que es una contracción... te diga a ti: "Tienes que guardar la calma, tienes que respirar y tal cosa” ... no, ¡no tiene ni idea!». Mujer profesional, asalariada, régimen contributivo.

«No hay como el suficiente tiempo para como asesorarlo, explicarle que opción es mejor, solamente uno llega y medio lo escuchan y ya recetan algo rápido y ya termino la consulta, no hay como esa atención, porque no es ese servicio que uno quisiera recibir como para despejar todas las dudas sino escasamente lo que uno medio siente así rápido y muchas veces ni lo miran a uno sino solo escriben en el computador y ya, no es más».

Mujer bachiller, trabajo informal, régimen subsidiado.

«Ellas me apoyaban incluso más que mi obstetra. Cuando él llegó y dijo no ya cesárea, pero las otras que pasaban, las obstetras de turno, cuando no estaba mi obstetra y me revisaban, me decían, vas súper bien, no te preocupes, y yo no, es que mi obstetra me dijo tal cosa, pero ellas me escuchaban, por lo menos me respondían, en cambio mi obstetra fue en la mañana, ya volvió después de almuerzo y ya cirugía».

Mujer profesional, ama de casa, plan complementario.

"Yo llegué con unas contracciones teniendo 33 semanas y 5 días, era para que me hubieran detenido las contracciones... Pero en ese entonces yo comía mucha papa y plátano, y estaba re gorda... yo no podía con mi peso, yo creo que subí como unos 20 kilos en ese embarazo, no manejaba una buena alimentación, entonces no tuve control, y cuando llegué, a ellos se les hizo fácil decir que estaba a término y me metieron a trabajo de parto».

Mujer profesional, asalariada, régimen contributivo. localidades más vulnerables de Bogotá y con pocas redes sociales (Tabla 1A), lo cual disminuye el uso de los servicios de salud. El dolor en el parto, en la lactancia materna, y con los procedimientos médicos, es naturalizado por el personal de salud y no existen alternativas para conectarse con el sentir de las mujeres.

\section{El saber-poder de las mujeres (Figura 1B)}

Las mujeres poseen saberes que han construido en sus historias de vida, a través de las redes sociales que las rodean y en los servicios de salud. La manera como se construyen estos saberes determina el poder y las decisiones que ellas toman en las instituciones de salud. Los aspectos relacionados con esta categoría se desarrollan en la Tabla 4.

Las desigualdades según clase social en los anteriores aspectos son: tener diferentes puntos de vista frente a una recomendación médica y derecho a quejarse o exigir para mejorar. En estos aspectos se afecta en mayor medida a las mujeres con una clase social menos aventajada. Al respecto, en la observación participante se evidenció que las participantes no 
Tabla 4. Códigos de la categoría el saber-poder de las mujeres

\begin{tabular}{|c|c|}
\hline Código & Definición conceptual \\
\hline $\begin{array}{l}\text { Presencia de } \\
\text { redes sociales }\end{array}$ & $\begin{array}{l}\text { No solo se refiere al apoyo que reciben las mu- } \\
\text { jeres de su compañero o la familia, sino tam- } \\
\text { bién de las redes de mujeres que se ayudan en- } \\
\text { tre sí, al personal de salud y agentes de salud } \\
\text { (doulas y parteras) que tejen alianzas y les dan } \\
\text { autonomía a las mujeres en la toma de deci- } \\
\text { siones de salud. }\end{array}$ \\
\hline
\end{tabular}

Prácticas institucionales naturalizadas

Resistencias en los servicios de salud

Derechos de las mujeres durante el embarazo, parto y posparto

Información dada en las instituciones

Independencia de la mujer
Se construyen históricamente y se normalizan en el proceso de atención. No son cuestionadas ni por el personal, ni por las mujeres y se ejercen bajo relaciones de poder donde las mujeres pierden autonomía en la toma de decisiones de salud.

Luchas que entablan las mujeres para tener autonomía en la toma de decisiones en salud. Por ejemplo, mentir al personal, ocultar información y no aceptar frontalmente las prácticas institucionales en salud.
Derechos que las mujeres conocen, negocian o exigen en los servicios de salud. Por ejemplo, tener diferentes puntos de vista frente a una recomendación médica, la necesidad de comprender los riesgos para interiorizar cuales se desean asumir y cuáles no y la necesidad de comprender los consentimientos informados y el plan de parto. Todo lo anterior les permite a las mujeres plantear inconformidades explícitamente y quejarse de la atención.

Información dadas en las instituciones de salud que les permite a las mujeres conocer sus derechos y mejorar la comprensión de los Procesos de Salud y Enfermedad (PSE).

Condiciones de género y clase social de las mujeres que les permite relacionarse con los ser-
Citas textuales

«Es afuera del sistema de salud donde uno tiene como que buscar las herramientas para poder empoderarse de lo que uno está sintiendo o de lo que uno sabe que puede hacer, entonces es la lectura, es ver las noticias, es el círculo de mujeres o de personas que están alrededor de uno que le pueden decir que sí se puede un parto natural. Lo anterior es contrario a la medicina porque ésta es como lo práctico para programar la agenda, la cesárea, y de pronto disminuir riesgos».

Mujer profesional, ama de casa, plan complementario.

"Yo aprendí en la clínica tradicional, pero tengo una voluntad de ir y entrenarse para hacer algo nuevo. A mí lo que me llama la atención es porque no hay la voluntad de ir a aprender nuevas técnicas en atención en parto y seguimos como anclados a lo de hace décadas, 40 años, no sé cuánto tiempo, me intriga el por qué no queremos salirnos».

Obstetra de medicina pre pagada.

«Me da miedo, es ese terror, por ejemplo yo ahorita estoy embarazada y estoy lactando a mi hijo todavía, de 28 meses y no le quiero decir a nadie si, entonces no quiero decirle a la enfermera porque me asusta que me diga algo, que me meta más miedo y pues yo estoy tranquila con lo que estoy haciendo y sé que lo que estoy haciendo no es un exceso tampoco, y sé que está controlado sí, pero si yo llego a decirle a alguien entonces va a ser la causante de muchas cosas, me van a regañar, me van a decir eso no está bien, tienes que dejarlo de hacer y si voy con mi esposo entonces voy a tener la presión de mi esposo entonces es como lo que no me gusta».

Mujer profesional, ama de casa, plan complementario. vicios de salud y tomar decisiones en el PSEA «que tienden los equipos de salud a ser lo que podríamos llamar «clasistas», se deja a la paciente que es de medicina prepagada, que está pagando, que paga mejores tarifas o que molesta más, se le brinda una mejor calidad, a la paciente del régimen contributivo que asiste a una institución de salud la atención va bajando y para la paciente del régimen subsidiado que se atiende porque toca y que es la que menos paga o la que menos puede molestar, se le puede brindar en algunos casos menos tiempo, menos atención, ... menos paciencia y pues eso va en contra totalmente de la atención que debemos tener con nuestras usuarias, que no deben tener ninguna diferencia por el aseguramiento para recibir la calidad médica». Obstetra régimen subsidiado.

«Volví con mi ginecólogo ese el que no me convence y estoy en la búsqueda de otro, yo quiero encontrar un equipo médico que trabaje bajo los principios del parto humanizado, pero digamos que no me ha sido fácil porque pues la información que ofrece el plan complementario es muy escasa, y no sé dentro de la institución médica como a quien dirigirme para preguntarle eso». Mujer profesional con especialización, trabajo informal, plan complementario.

«No tengo miedo como al comienzo de pronto con mi embarazo anterior de sentirme presionada por mi esposo primero, porque igual el terror que nos metió el obstetra fue a los dos, entonces obviamente cuando vas con tu esposo, tu esposo también te está mirando y te dice tenemos que hacerlo porque es que está en riesgo la vida de nuestro hijo. Esta vez simplemente es mi cuerpo y voy a estar un poquito más segura y si yo decido que no voy a ceder a la cesárea, no lo voy a hacer, y si yo decido que voy a cambiar de obstetra las veces que yo considere necesario, lo voy a hacer».

Mujer profesional, ama de casa, plan complementario. manifestaban frontalmente las inconformidades referentes a la atención prestada, ni tampoco se estructuraban quejas formales en los servicios de salud. Al dialogar con las mujeres sobre este aspecto, manifestaban miedo a la confrontación y los derechos adquirían una connotación de favor en las instituciones públicas. Igualmente, en estos servicios existe un déficit de insumos y talento humano reduciendo las alternativas de elección de personal capacitado en prácticas humanizadas.

\section{DISCUSIÓN}

Los hallazgos encontrados evidencian cambios en la relación médico-paciente, teniendo en cuenta que esa interacción es una relación social, intersubjetiva y resultado del contexto sociopolítico en la cual se desarrolla. Existen factores estructurales que determinan el funcionamiento de los sistemas de salud bajo el concepto de oferta y demanda en el mercado mundial ${ }^{(25)}$. En la relación 
médico-paciente se integran elementos tecnológicos como el uso del computador, que es relevante para la inscripción de los datos y adquiere un valor por encima de la interacción de los sujetos. Igualmente es visible la relación con el poder del sector administrativo que disminuye la libertad del profesional, para responder a la economía de mercado, subordinando la ética del personal de salud ${ }^{(25)}$. En esta investigación esta problemática hace parte de las barreras estructurales que tienen las instituciones para aplicar las prácticas humanizadas en el EPP.

En ese contexto no es importante la relación entre sujetos sino los resultados que se esperan del acto médico. La tecnología también se transforma pues ya no es una ayuda, sino que es central en donde se estructura la medicina. Todo lo anterior es más importante que el enfermo y la labor médica se deshumaniza en un contexto sociopolítico que alimenta esta problemática ${ }^{(25)}$. En esta investigación la tecnología adquiere un valor más importante que la clínica y que el acompañamiento cuando se trata de un parto fisiológico y natural.

La relación médico-paciente se elimina por la agenda administrativa institucional que es más importante que la agenda del médico y del paciente. En ese contexto se despersonaliza la atención y se automatiza el encuentro. La agenda del médico se ve estructurada a partir de las condiciones espacio-temporales de la consulta médica. El espacio y el tiempo de esta consulta lo define la institución desde un marco administrativo que busca hacer la gestión médica rentable. El médico llega un espacio para el cual se prepara en su formación médica y se ve obligado a aplicar sus conocimientos biomédicos en condiciones difíciles, pues el tiempo de consulta y las actividades administrativas que toca realizar por orden institucional eliminan la posibilidad de tener en cuenta los aspectos socio culturales del proceso salud enfermedad (PSE) ${ }^{(26)}$.

Referente a las identidades masculinas y femeninas, la medicina ha sido masculinizada históricamente. Esto se visualiza en los discursos de la sociedad médica inglesa en el siglo XVII, donde se les aconseja a los profesionales evitar el contacto y la intimidad con los pacientes. En el siglo XIX, se identifica en la sociedad victoriana cómo el concepto de simpatía (entendida como afinidad entre personas que se atraen entre sí) se considera femenino y no científico, al igual que las relaciones entre médico y paciente. En Estados Unidos posterior a la graduación de médicas, algunas de ellas integraron en su quehacer la simpatía, sin embargo, otras abogaron más por la tecnología y estar separadas de las relaciones interpersonales. Estos aspectos han influido para que en el siglo XX, la simpatía definida como habilidades de carácter afectivo también perdiera su valor científico ${ }^{(27)}$.

El desconocimiento de la historia de la profesión médica permite que en la actualidad esta masculinización perdure y sean entendibles las dificultades para integrar conocimiento teórico-práctico y la curación-cuidado ${ }^{(27)}$, lo cual afecta la atención en salud de las usuarias de los servicios de salud, pues estas transitan en instituciones de salud donde el personal trabaja individualmente.

La violencia en contra de las mujeres en EPP tiene sus orígenes en la formación médica, donde existen currículos ocultos que se aprenden históricamente en los ámbitos académicos. Existe un habitus desvinculante por parte del personal de salud que ignora el carácter humano de la mujer durante el parto, por eso es posible que se validen comportamientos irrespetuosos. El habitus autoritario genera amenazas contra las mujeres que no siguen las órdenes médicas, las culpabilizan cuando no «colaboran» (llamado a la norma) en el trabajo de parto y descalifican el dolor que sienten. En el habitus en acción, se ve a las mujeres como inferiores desde el punto de vista profesional y de género, lo cual es relevante para que funcionen las reglas represivas que las mujeres aceptan de manera subordinada en un contexto donde se supone se merece el sufrimiento y se ignora la realidad social de la población ${ }^{(28)}$.

El género atraviesa las instituciones sociales ${ }^{(29)}$ y los servicios de salud no son la excepción ${ }^{(30)}$. En ellos se instauran los roles femeninos y masculinos que se relacionan, dando cuenta de las jerarquías desarrolladas a nivel social ${ }^{(29,30)}$. Los compañeros de las mujeres, presentes en la investigación, son excluidos del proceso de atención, adjudicando el rol reproductivo exclusivo a la mujer. Sin embargo, los hombres se incluyen en los servicios cuando se desean imponer ciertas prácticas institucionales sobre las mujeres. La autonomía en la toma de decisiones sobre el cuerpo de la mujer está determinada por la presencia del hijo, sobre el cual juegan papeles importantes el estado, los profesionales de la salud y el padre del feto.

Dentro de las limitaciones, esta investigación no buscó generalizar como tradicionalmente lo hacen los estudios poblacionales, sino comprender aspectos específicos frente a la problemática planteada en la investigación. No se hizo una etnografía tradicional donde la investigadora convive permanentemente en el lugar de trabajo de campo, pero se realizó 13 meses de observación, permaneciendo en el terreno mínimo tres días a la semana. La observación participante fue realizada solo por la investigadora principal teniendo en cuenta limitaciones económicas. Esta investigadora no es antropóloga, sino médica bogotana, quien hace parte del contexto, y su visión podría ignorar otros aspectos que pudieran ser relevantes en el análisis de la información. Sin embargo, esta investigación en salud pública ha sido un trabajo colaborativo, donde fue relevante el apoyo de investigadores de las ciencias sociales, quienes ayudaron a construir una visión integral de los asuntos médicos que se quieren analizar. Hubiera sido posible que participara un mayor número de personas en busca de la saturación de la información con relación a la clase social, pero dado el presupuesto y el cronograma establecido no se invitó a participar a más mujeres. 
En conclusión, se hace necesario el desarrollo de intervenciones en instituciones educativas y de salud que tengan en cuenta las propuestas teóricas y metodológicas de esta investigación y la democratización de la información médica. Las desigualdades e inequidades en salud son prevenibles a través de políticas públicas con enfoque de género que contribuyan a transformar ambos, el sistema de salud y educativo colombiano. Se requiere profundizar en la afectación del PSE por la falta de calidad en el proceso de atención e identificar las diferentes masculinidades que juegan roles importantes en la relación de las mujeres con el personal.

Agradecimientos: A las mujeres y al personal de salud que participaron en la investigación. Fundación Arka, Organización Apapachoa, Movimiento Nacional por la Salud Sexual y Reproductiva en Colombia, Tribu Criarte, Secretaría de Salud Bogotá. A Marcelo Amable, Alfredo

\section{REFERENCIAS BIBLIOGRÁFICAS}

1. Downe S, Lawrie TA, Finlayson K, Oladapo OT. Effectiveness of respectful care policies for women using routine intrapartum services: a systematic review. Reproductive health. 2018;15(1):23. doi: 10.1186/ s12978-018-0466-y

2. Organización Mundial de la Salud. Prevención y erradicación de la falta de respeto y el maltrato durante la atención del parto en centros de salud [Internet] Ginebra OMS; 2014 [citado 30 deEnero de 2020]. Disponible en:https:// apps.who.int/iris/bitstream/handle/10665/134590/WHO_RHR_14.23_spa. pdf;sessionid=8D5556D17EF46062AA6DDB21F34094F4? sequence=1.

3. Monroy MSA. El continuo ginecobs-tétrico .Experiencias de violencia vividas por mujeres gestantes en servicios de salud en Bogotá [Tesis maestría]. Bogotá: Escuela de estudios de Genero Universidad Nacional de Colombia; 2012.Disponible en: http://www.bdigital.unal.edu.co/7805/1/ soniaandreamonroymu\%C3\%B1oz.2012.pdf

4. Briceño Morales X, Enciso Chaves LV, Yepes Delgado CE. Neither Medicine Nor Health Care Staff Members Are Violent By Nature: Obstetric Violence From an Interactionist Perspective. Qualitative health research. 2018;28(8):1308-19. doi: 10.1177/1049732318763351

5. Vallana Sala VV. Parirás con dolor, lo embarazoso de la práctica obs-tétrica. Discursos y prácticas que naturalizan la violencia obstétrica en Bogotá [Tesis de maestría]. Bogotá: Facultad de Ciencias Sociales. Pontificia Universidad Javeriana; 2016. Disponible en: https://repository.javeriana. edu.co/bitstream/handle/10554/19135/VallanaSalaVivianaValeria2016. pdf? sequence $=1$ \&isAllowed $=y$

6. Colón IC. Sentimientos, memorias y experiencias de las mujeres en trabajo de parto el caso de centros hospitalarios en Cartagena [Tesis de maestría]. Cartagena: Escuela de estudios de Genero Universidad de Cartagena y Nacional de Colombia; 2008. Disponible en: http://bdigital.unal.edu. co/53287/1/candelariacoloniriarte.2008.pdf

7. Rocha-Acero ML, Socarrás-Ronderos F, Rubio-León DC. Prácticas de atención del parto en una institución prestadora de servicios de salud en la ciudad de Bogotá. 2019;37(1):53-65. doi: 10.17533/udea.rfnsp.v37n1a10

8. Jojoa-Tobar E, Cuchumbe-Sánchez YD, Ledesma-Rengifo JB, Muñoz-Mosquera MC, Suarez Bravo JP. Violencia obstétrica: haciendo visible lo invisible. Rev Univ Ind Santander Salud. 2019;51(2):136-47. doi: http:// dx.doi.org/10.18273/revsal.v51n2-2019006

9. Bedoya-Ruiz LA, Agudelo-Suárez AA. Relación de las mujeres en embarazo, parto y postparto (EPP) con los servicios de salud según la clase social. Rev Gerenc Polít Salud. 2019;18(36). doi: 10.11144/Javeriana. rgps18-36.rmep

10. Breilh J. Las categorías causalidad y clase social como elementos de la ideología epidemiológica. En: Breilh J, editor. Crítica a la interpretación
Maya y Mónica Saenz por sus aportes desde las ciencias sociales. A la Universidad CES de Medellín y Colciencias por la financiación.

Contribuciones de los autores: Todos los autores participaron en la concepción, el diseño del trabajo, análisis, interpretación de datos, revisión crítica y aprobación de la versión final. LABR realizó la recolección de datos y la escritura inicial del artículo. Todos los autores se hacen responsables y garantes de todos los aspectos que integran el manuscrito. Este artículo es parte del proceso de formación doctoral de LABR en el Doctorado en Salud Pública de la Universidad CES y será usado como parte del material empleado para la disertación de la tesis.

Fuentes de financiamiento: Beca doctorado nacional Colciencias convocatoria 647-2014 y convocatoria de pequeña cuantía de la Universidad CES de Medellín, Colombia.

Conflictos de interés: Todos los autores declaran que no tienen conflicto de interés alguno. capitalista de la Epidemiología Un ensayo de desmitificación del proceso de salud y enfermedad. Méjico: Universidad Autonoma Metropolitana; 1977. P. 78-90.

11. Breilh J. Breve recopilación sobre operacionalización de la clase social para encuestas en la investigación social. En: Centro de estudios y asesoría en salud (CEAS), editor. Quito: Ecuador Centro de estudios y asesoría en salud (CEAS); 1989. p. 1-12.

12. Menéndez EL. El modelo médico hegemónico. Estructura función y crisis. En: Menéndez EL, editor. Morir de Alcohol: Saber y Hegemonía Médica. Mexico DF: Alianza Editorial Mexicana; 1990. p. 83-117.

13. Menéndez E.L. El punto de vista del actor: homogeneidad, diferencia e historicidad. En: Menéndez E.L, editor. La parte negada de la cultura: relativismo, diferencias y racismo. Barcelona: Edicions Bellaterra; 2002. p. 291-365.

14. Grondin J. Que es la interpretación. En: Grondin J, editor. El Legado de la Hermeneutica Primera ed. Cali: Universidad del Valle; 2009. p. 15-36.

15. Packer M. La investigación enmancipadora como reconstrucción racional En: Packer M, editor. La ciencia de la investigación cualitativa. Bogota, Universidad de los Andes: Facultad de Ciencias Sociales; 2013. p. 337-363.

16. Kincheloe J, McLaren P. Replanteo de la teoria critica y de la investigación cualitativa. En: Denzin NK, Lincoln YS, editores. Manual de investigación cualitativa Volumen II Paradigmas y perspectivas en disputa. Barcelona: Gedisa; 2012. p. 241-91.

17. Scheper-Hughes N. Nervoso. En: Scheper-Hughes N, editor. La muerte sin llanto Violencia y vida cotidiana en Brasil. Barcelona: Ariel; 1997. p. 167-212.

18. Mendizabal N. Los componenentes del diseño flexible en la investigación cualitativa. En: Vasilachis de Gialdino, editor. Estrategias de investigación cualitativa. Barcelona: Gedisa; 2006. p. 65-103.

19. Galeano ME. Diseño de proyectos en la investigación cualitativa: Fondo Editorial Universidad EAFIT; 2004. p. 11-81.

20. Organización Panamericana de la Salud. Perfil sistema de salud en Colombia. [Internet] Washington, D.C: Organización Panamericana de la Salud; 2010 [citado 30 de enero de 2020]. Disponible en : https://www.paho.org/ hq/dmdocuments/2010/Perfil_Sistema_Salud-Colombia_2009.pdf?ua=1

21. Restrepo ODA. Vigencia de la categoría clase social en salud pública. En: Estrada M J H, editor. Teoria Critica de la Sociedad y Salud Publica. Bogotá: Universidad Nacional de Colombia. Universidad de Antioquia; 2011.p. 134-44.

22. Fontana A, Frey JH. La entrevista. En: Denzin N, Lincoln Y, editores. Manual de Investigación Cualitativa Volumen IV Métodos de recolección y análisis de datos. Barcelona: Gedisa, 1ª edición; 2015. p. 140-189. 
23. Evens TMS, Handelman D. Introduction: The Ethnographic Praxis of the Theory of Practice. Social Analysis [Internet] Brooklyn NY: The International Journal of Social and Cultural Practice; 2005 [citado el 30 de Enero de 2020]. Disponible en : www.jstor.org/stable/23179071

24. Strauss A, Corbin J. Bases de la investigación cualitativa. Técnicas y procedimientos para desarrollar la teoría fundamentada. Medellin: Editorial Universidad de Antioquia Facultad de Enfermería de la Universidad de Antioquia; 2002 .p. 3-197

25. Rossi I. Capítulo 2. La clínica como espacio social. Época de cambios o cambio de época?. En: Hamui Sutton L, Maya A P, Hernández Torres I, editores. La comunicación dialógica como competencia médica esencial. Ciudad de Mexico: Manual Moderno; 2018. p. 38-58.

26. Cruz Sanchez M, Hernández Torres I, Grijalva MG, Maya A P, Dorantes P. Capitulo 3. El ejercicio de la profesión médica y la comunicación médico paciente en contextos situacionales. En: HamuiSutton L, Maya A P, Hernández Torres I, editores. La comunicación dialógica como competencia médica esencial. Ciudad de Mexico: Manual Moderno; 2018. p. 58-100.
27. Ortiz G T. El género, organizador de profesiones sanitarias. En: Miqueo C, Tomas C, Tejero C, Barral M J, Fernandez T, Yago T, editors. Perspectivas de Género en Salud Fundamentos Científicos y Socioprofesionales de Diferencias Sexuales No Previstas. Madrid: Minerva Ediciones, S.A; 2001. p. 53-77.

28. Castro R. El habitus en acción. La atención autoritaria del parto en los hospitales. En: Castro R, Ervite J editores. Sociología de la práctica médica autoritaria. Violencia obstetrica, anticoncepción inducida y derechos reproductivos. Cuernavaca, Mexico: Universidad Nacional Autonoma de Mexico; 2015. p. 81-131.

29. Connell R, Pearse R. Gender theorist and gender theory. En: Connell R, Pearse R, editors. Gender In world perspective 3rd ed. Cambridge: Polity Press; 2015. p. 68-206.

30. Lorber J, Moore LJ. Hierarchies in helath care: patients, professionals and gender. En: Lorber J, Moore LJ, editors. Gender and the social construction of illnes. Lanham: Altamira Press; 2002. p. 37-51.

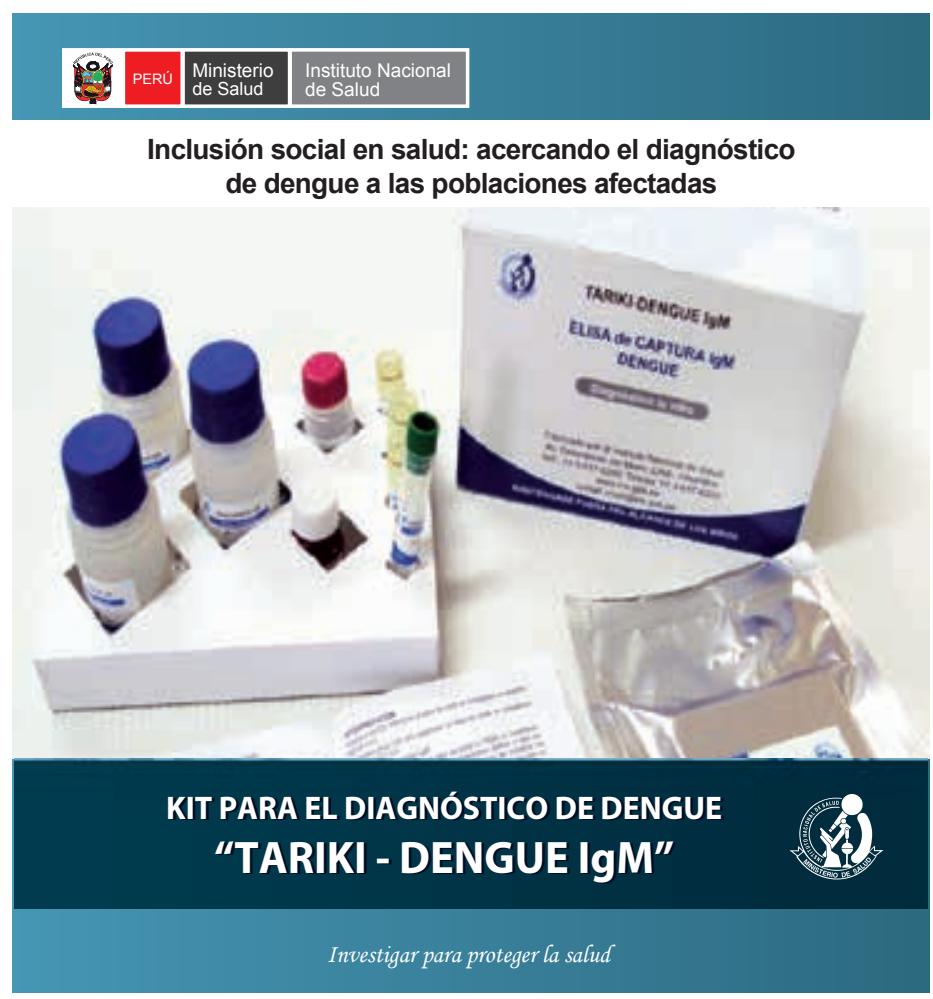

